\title{
The $(N=1)$ Supersymmetric Sine-Gordon Model in Two Dimensions. I
}

\author{
Marzio Cassandro ${ }^{1}$, Francesco Nicolò ${ }^{2}$, and Benedetto Scoppola ${ }^{3}$ \\ ${ }^{1}$ Dipartimento di Fisica, Università della Sapienza, C.N.R.-GNSM-C.N.R.-GNFM, Roma, Italy \\ ${ }^{2}$ Dipartimento di Fisica, Università di Lecce, Lecce, Italy \\ ${ }^{3}$ I.N.F.N., Sezione di Roma, Italy
}

\begin{abstract}
In this paper and its companion (II) we prove that the Supersymmetric $N=1$ massless Sine-Gordon field theory, at finite (space) volume, exists and is analytic in the coupling constant $\lambda$. Moreover at finite (space) volume is Witten index is $=1$.
\end{abstract}

\section{Table of Contents}

Introduction . . . . . . . . . . . . . . . . . . . . . . . . . . . . 681

1. The Supersymmetric Model: Definitions and Notations . . . . . . . . . . . 682

2. The Euclidean Continuation: Regularizations and Definition of the Interacting Measure . . . . . . . . . . . . . . . . . . . . . . . . . . . 684

3. The Introduction of the Wick product . . . . . . . . . . . . . . . . 689

4. The Effective Potential . . . . . . . . . . . . . . . . . . . . . . 690

5. The Results: The Existence and the Analyticity of the Theory . . . . . . . . . 691

6. The Lattice Regularization and O.S. Positivity . . . . . . . . . . . . . . 693

7. The Results: The Supersymmetry is not Spontaneously Broken . . . . . . . . 696

8. The Strategy of the Proofs . . . . . . . . . . . . . . . . . . . . . 696

9. Some Remarks on the Scalar Sine-Gordon Theorey. . . . . . . . . . . . 697

10. Conclusions . . . . . . . . . . . . . . . . . . . . . . . . . . .698

\section{Introduction}

Supersymmetry has had in recent years a relevant impact in field theory both on the physical and on the mathematical side. Specifically a question which has deep connections with modern mathematics is the presence or not of a spontaneous breaking of the supersymmetry in some field theory models (Witten [1]). This question is connected to the study of the index of an operator (a Dirac operator in a infinite dimensional space). All this, apart from more phenomenological aspects, makes it interesting to perform a rigorous mathematical study of the supersymmetric field theory models. This problematic is thoroughly discussed in the papers of Jaffe et al. [2] where they study the $N=1$ and $N=2$ Wess-Zumino models. 
Here we rigorously study another supersymmetric two dimensional model: the supersymmetric $N=1$ massless Sine-Gordon model. We prove the existence (from the constructive point of view) of the theory in a finite (spatial) volume for a certain range of values of its parameters $\lambda$ and $\alpha$, the coupling and the wave function constants of the model, respectively, in the limit of the ultraviolet cutoff going to infinity. Moreover for a fixed (spatial) volume, the theory is analytic in the coupling constant $\lambda$ in a disk of the complex $\lambda$ plane around the origin whose radius depends on the volume (it shrinks to zero as the volume tends to infinity). This means that the perturbation series for the Schwinger functions or for the expectation of any observable of the theory are convergent. Finally we prove that the supersymmetry of this model is not spontaneously broken.

These results are obtained using some "Renormalization Group techniques" developed some years ago by Gallavotti and collaborators [3] to study the $\Phi_{3}^{4}$ euclidean quantum field theory and using an expansion, again based on the Renormalization group, called the "tree expansion" introduced by Gallavotti and one of the authors (F.N.) [4] to study from this modern point of view the renormalization of the non-superrenormalizable field theories.

The strategy of this work is the following one: we first introduce a regularized version of our model that preserves supersymmetry and shows that the tree expansion is convergent uniformly in the cutoff. The proof follows the strategy introduced in $[14,16]$ to study the scalar case. Some modifications are needed due to the presence of the fermions and to the fact that the free theory is massless. Unfortunately the regularized theory we work with is not Osterwalder-Schrader (O.S.) positive and we have not been able to find any other regularization sharing the three following properties:

i) Supersymmetry

ii) O.S. positivity

iii) Exponential decay of the covariances both in space and time that we require as a crucial technical tool to prove, using the renormalization group, the convergence of the perturbative series.

The next step then is to consider a lattice version of our model, where we lose supersymmetry invariance but we gain O.S. positivity and we preserve the exponential decay properties of the covariances, so that it is possible to apply with minor changes the previous analysis and prove that also in this case the tree expansion is uniformly convergent in the cutoff, i.e. now the lattice spacing. The uniform convergence allow us to remove explicitly the cutoff in both cases and to show that in this limit the Schwinger functions of the two theories are equal order by order in perturbation theory. Therefore the limit theory is unique and exhibits at the same time O.S. positivity and supersymmetry.

\section{The Supersymmetric Model: Definitions and Notations}

There are many ways of defining a supersymmetric field theory. We will use the superfield formalism. We start with some definitions. 
As we are interested in the $N=1$ theory, the spinorial anticommuting parameters are all real,

$$
\begin{gathered}
\xi^{\alpha}=\varepsilon^{\alpha \beta} \xi_{\beta}, \\
\varepsilon^{\alpha \beta}=-\left(\begin{array}{cc}
0 & 1 \\
-1 & 0
\end{array}\right), \\
(\xi \eta)=\xi^{\alpha} \eta_{\alpha}=\varepsilon^{\alpha \beta} \xi_{\beta} \eta_{\alpha} .
\end{gathered}
$$

We make the following choices of $\gamma$ matrices:

$$
\begin{aligned}
& \gamma^{0}=\left(\begin{array}{cc}
0 & i \\
-i & 0
\end{array}\right), \quad \gamma^{1}=\left(\begin{array}{ll}
0 & i \\
i & 0
\end{array}\right), \\
& \left\{\gamma^{\mu}, \gamma^{\nu}\right\}=2 g^{\mu \nu}, \quad g^{00}=-g^{11}=1 .
\end{aligned}
$$

We define the scalar superfield (see Wess-Bagger [5], for a more complete discussion of the mathematical aspects of the Superfield formalism see [17]):

$$
\Phi(x, \theta)=\phi(x)+i \theta \cdot \psi(x)+\frac{i}{2}(\theta \cdot \theta) F(x)
$$

where $F(x)$ is the so called "auxiliary field" (real), $\phi(x)$ is a real scalar field and $\psi_{\alpha}$ is a Maiorana field (real), $(\alpha=1,2)$.

In the superspace $(x, \theta)=\left(x^{0}, x^{1} ; \theta^{1}, \theta^{2}\right)$ we introduce the following covariant derivatives:

$$
Q_{\alpha}=D_{\alpha}=\frac{\partial}{\partial \theta^{\alpha}}+\left(\gamma^{\mu} \theta\right)_{\alpha} \partial_{\mu}, \quad \bar{Q}_{\alpha}=\bar{D}_{\alpha}=i \varepsilon^{\alpha \beta} D_{\beta}=\left(\gamma^{0} D\right)_{\alpha},
$$

and their conjugate ones

$$
\mathscr{P}_{\alpha}=\frac{\partial}{\partial \theta^{\alpha}}-\left(\gamma^{\mu} \theta\right)_{\alpha} \partial_{\mu}, \quad \overline{\mathscr{P}}_{\alpha}=i \varepsilon^{\alpha \beta} P_{\beta}=\left(\gamma^{0} P\right)_{\alpha}
$$

The covariant derivatives $D_{\alpha}, \bar{D}_{\alpha}$ are a realization of the superalgebra on the superspace; in fact defining

$$
P_{\mu}=-i \partial_{\mu},
$$

we obtain

$$
\left\{Q_{\alpha}, \bar{Q}_{\beta}\right\}=2\left(\gamma^{\mu} P_{\mu}\right)_{\alpha \beta}, \quad\left[Q_{\alpha}, P_{\mu}\right]=0, \quad\left[\bar{Q}_{\alpha}, P_{\mu}\right]=0,
$$

which are the commutation and anticommutation relations of the $N=1$ graded Lie algebra. Moreover

$$
\left\{Q_{\alpha}, \mathscr{P}_{\beta}\right\}=0, \forall \alpha, \beta \text {. }
$$

The supersymmetric action of the free theory is

$$
\begin{aligned}
\mathscr{A}_{0} & =\frac{-i}{4} \int d^{2} x d^{2} \theta\left[\Phi(x, \theta)\left(\mathscr{P}^{\alpha} \mathscr{P}_{\alpha}\right) \Phi(x, \theta)\right]=-i \int d^{2} x \mathscr{L}_{\text {free }}(\phi, \psi, F) \\
& =\frac{-i}{2} \int d^{2} x\left[\dot{\phi}^{2}-\left(\partial_{1} \phi\right)^{2}+\bar{\psi}\left(i \gamma^{\mu} \partial_{\mu}\right) \psi+F^{2}\right] .
\end{aligned}
$$


The interacting part of the action is:

$$
\mathscr{A}_{1}=-\int d^{2} x d^{2} \theta[\lambda \cos \alpha \Phi(x, \theta)]
$$

and, in the Minkowski space, the total action is:

$$
\begin{aligned}
\mathscr{A}= & \mathscr{A}_{0}+\mathscr{A}_{1}=\frac{-i}{2} \int d^{2} x\left\{\left[\dot{\phi}^{2}-\left(\partial_{1} \phi\right)^{2}+\bar{\psi}\left(i \gamma^{\mu} \partial_{\mu}\right) \psi+F^{2}\right]\right. \\
& \left.+\left[\lambda \alpha^{2} \bar{\psi} \psi \cos \alpha \phi-2 \lambda \alpha F \sin \alpha \phi\right]\right\} \\
= & \frac{-i}{2} \int d^{2} x\left\{\left[\dot{\phi}^{2}-\left(\partial_{1} \phi\right)^{2}+\bar{\psi}\left(i \gamma^{\mu} \partial_{\mu}\right) \psi+F^{2}\right]\right. \\
& \left.+\left[-\bar{\psi} \psi\left(\frac{\partial^{2} W_{0}}{\partial \phi^{2}}\right)+2 F\left(\frac{\partial W_{0}}{\partial \phi}\right)\right]\right\}
\end{aligned}
$$

where

$$
W_{0}=\lambda \cos \alpha \phi \text {. }
$$

If we consider the supersymmetric massive theory we just have to replace $W_{0}(\phi)$ with

$$
W(\phi)=\lambda \cos \alpha \phi+\frac{1}{2} m \phi^{2} .
$$

We will call the model we are investigating "massless" in the sense that no supersymmetric mass term is present in the Lagrangian; this of course will not exclude the possibility of dynamically generating a mass [11].

All these definitions are formal. In the next section we write the corresponding formal action in the euclidean formalism and, introducing the appropriate regularizations, we give a rigorous meaning to it.

\section{The Euclidean Continuation, Regularization and Definition of the Global Measure}

A standard procedure to study the field theory described by the action introduced in Eq. (1.14) is the so-called Euclidean continuation. This is achieved with the following substitution:

$$
\begin{gathered}
t \rightarrow i \tau, \quad \gamma^{0} \rightarrow \gamma^{0 E}=\gamma^{0}, \\
\left(x^{1}, t\right) \rightarrow\left(x^{1}, \tau\right), \quad \gamma^{1} \rightarrow \gamma^{1 E}=i \gamma^{1}, \\
\phi\left(x^{1}, t\right) \rightarrow \phi_{E}\left(x^{1}, \tau\right), \quad \psi\left(x^{1}, t\right) \rightarrow \psi_{E}^{(1)}\left(x^{1}, \tau\right), \\
\frac{\partial}{\partial t} \phi\left(x^{1}, t\right) \rightarrow-i \frac{\partial}{\partial \tau} \phi_{E}\left(x^{1}, \tau\right), \quad \bar{\psi}\left(x^{1}, t\right) \rightarrow \psi_{E}^{(2)}\left(x^{1}, \tau\right), \\
F\left(x^{1}, t\right) \rightarrow-i F_{E}\left(x^{1}, \tau\right),
\end{gathered}
$$

where in general $\psi_{E}^{(1)}, \psi_{E}^{(2)}$ are two independent fermionic Grassmann fields. This is usually called the "Fermi fields doubling" (see Osterwalder and Schrader [6]). The explicit expression of these Euclidean fields and their connection with the Minkowski ones are given in Appendix A. 
With the substitutions (2.1), (2.2) we can write, still formally, the following Euclidean path integral for a transition amplitude at imaginary times:

$$
\begin{aligned}
\left\langle f\left|e^{-H\left(\tau_{f}-\tau_{l}\right)}\right| i\right\rangle= & \mathscr{N}^{-1} \int \mathscr{D} \phi_{E} \mathscr{D} F_{E} \mathscr{D} \psi_{E} e_{f}\left(\phi_{E}, \psi_{E}, F_{E}\right) e_{i}\left(\phi_{E}, \psi_{E}, F_{E}\right) \exp \frac{-1}{2} \int d^{2} x \\
& \times\left\{\left[\left(\partial_{\mu} \phi_{E}\right)^{2}+\psi_{E}^{(2)}\left(-\gamma^{\mu E} \partial_{\mu}\right) \psi_{E}^{(1)}+F_{E}^{2}\right]\right. \\
& \left.+\left[-\psi^{(2) E} \psi_{E}^{(1)} \lambda \alpha^{2} \cos \alpha \phi_{E}-2 i \lambda \alpha F_{E} \sin \alpha \phi_{E}\right]\right\}
\end{aligned}
$$

where $H$ is the Hamiltonian of our system and $|f\rangle$ and $|i\rangle$ two arbitrary final and initial states. On the right-hand side of Eq. (2.3) everything is expressed in terms of "Euclidean objects". $e_{f}$ and $e_{i}$ are the wave functionals of the final and initial states. $\mathscr{D} \phi_{E}$ and $\mathscr{D} F_{E}$ are defined formally as

$$
\mathscr{D} \phi_{E}=\prod_{x} d \phi_{E}(x) ; \quad \mathscr{D} F_{E}=\prod_{x} d F_{E}(x)
$$

$\mathscr{D} \psi_{E}$ will be defined in a moment. of

Looking at Eq. (2.3) we see that the right-hand side is, formally, the integration

$$
\exp V\left(\phi_{E}, \psi_{E}^{(1)}, \psi_{E}^{(2)}, F_{E}\right)=\exp \frac{-1}{2} \int d^{2} x\left[\psi_{E}^{(2)} \psi_{E}^{(1)} \lambda \alpha^{2} \cos \alpha \phi_{E}+2 i F_{E} \lambda \alpha \sin \alpha \phi_{E}\right]
$$

with respect to a measure which is a product of a gaussian measure for the field $\phi_{E}$, a gaussian measure for the auxiliary field $F_{E}$ and a measure with respect to the fermionic fields $\psi_{E}^{(1)}, \psi_{E}^{(2)}$. Notice that in Eq. (2.2) $F$ was continued to $i F_{E}$ to make convergent the integration with respect to $F$ (see Nicolai [7]).

In [6] it has been proven that it is possible to define two fermionic fields $\psi_{E}^{(1)}$, $\psi_{E}^{(2)}$ expressed in terms of anticommuting creation and destruction operators in a Euclidean Fock space such that:

$$
\begin{gathered}
\left\langle\psi_{E, \alpha}^{(1)}(x) \psi_{E, \beta}^{(2)}(y)\right\rangle_{E}=\left\langle T\left(\hat{\psi}(x)_{M, \alpha} \hat{\psi}(x)_{M, \beta}\right)\right\rangle_{M}=S_{\alpha, \beta}(x, y), \\
S_{\alpha, \beta}(x, y)=\frac{1}{(2 \pi)^{d}} \int d^{d} p \frac{e^{-i p(x-y)}\left(m-i p^{E}\right)_{\alpha, \beta}}{p^{2}+m^{2}},
\end{gathered}
$$

where $\hat{\psi}(x)_{M}$ is the free fermionic field, in the Minkowski formalism, at imaginary times. $T$ denotes the time ordered product and $\langle.\rangle_{M}$ is the vacuum expectation of the Minkowski fermionic Fock space. $\langle.\rangle_{E}$ is the vacuum expectation in the Euclidean fermionic Fock space. Nicolai in [7] has discussed the modifications one has to introduce when $\psi_{M}$ is a Maiorana spinor instead of a generic Dirac one. In this case the following relation holds:

$$
\psi_{E, \alpha}^{(2)}=\left(C \psi_{E}^{(1)}\right)_{\alpha}
$$

Nevertheless there is still a doubling of the fermionic degrees of freedom as in the Minkowski space $\psi_{M}(x)=\psi_{M}^{+}(x)$ while this is not true for $\psi_{E}^{(i)}$.

In the two dimensional case $\psi_{E}^{(i)}$ are two component spinors and with our choice of the $\gamma$ matrices:

$$
C=\gamma^{0} \text {. }
$$


Finally it is well known that one can represent the anticommuting operators in the holomorphic representation (Faddeev and Slavnov [8]) where they are generators of an infinite Grassmann algebra. In this framework we can write

$$
\psi_{E, \alpha}^{(i)}(x)=\frac{1}{(2 \pi)^{2}} \int d^{2} p e^{i p x} \hat{\psi}(p),
$$

where $\hat{\psi}_{\alpha}(p)$ is an anticommuting Grassmann variable,

$$
\mathscr{D} \psi_{E}=\prod_{p} d \hat{\psi}_{E}^{(1)}(p) d \hat{\psi}_{E}^{(2)}(p)
$$

and prove that, formally

$$
S_{\alpha, \beta}(x, y)=\mathscr{N}_{\psi}^{-1} \int \mathscr{D} \psi_{E}\left[\exp \int d^{2} x \psi_{E}^{(2)}\left(\gamma^{\mu E} \partial_{\mu}\right) \psi_{E}^{(1)}\right] \psi_{E, \alpha}^{(1)}(x) \psi_{E, \beta}^{(2)}(y) .
$$

This setting fixed, we can give a rigorous definition of the measure of the interacting theory introducing the appropriate regularizations.

The Regularization of the Model. We start observing that Eq. (2.3) is correct at the formal level, that is disregarding the regularizations, although the auxiliary field $F(x)$ is not a canonical variable because its conjugate momentum is absent. In fact integrating with respect to $F_{E}$ the right-hand side of $(2.3)$ we obtain the same expression we would obtain quantizing directly the classical Lagrangian (1.14) with the field $F$ eliminated using the classical equations of motion and then reexpressing the transition amplitude in the path integral formalism.

The reason we prefer to keep $F_{E}$ is that this allows us to regularize the theory in a obvious supersymmetric way and moreover the regularized fields will transform exactly in the same way as the unregularized ones. Therefore our procedure will be to regularize simultaneously and in the same way the fields $\phi, \psi, F$. This procedure is equivalent to choosing a well defined regularization for the Lagrangian expressed as a function of the fields $\phi$ and $\psi$ only.

Fixed this regularization strategy, to regularize the model we have to introduce:

a) an ultraviolet cutoff,

b) a "regularization" due to the fact that we are considering a massless theory in two dimensions,

c) finite volume boundary conditions.

a) Disregarding for the moment points b) and c), the regularized covariances of a massive theory in an infinite volume can be defined in the following way:

$$
\begin{aligned}
& C^{[\leqq N]}(x, y)=\frac{1}{(2 \pi)^{2}} \int d^{2} p e^{i p(x-y)} \frac{1}{p^{2}+m^{2}} \exp \frac{-\left(p^{2}+m^{2}\right)}{\gamma^{2 N}}, \\
& S^{[\leqq N]}(x, y)=\frac{1}{(2 \pi)^{2}} \int d^{2} p e^{i p(x-y)} \frac{i p^{E}+m}{p^{2}+m^{2}} \exp \frac{-\left(p^{2}+m^{2}\right)}{\gamma^{2 N}}, \\
& D^{[\leqq N]}(x, y)=\frac{1}{(2 \pi)^{2}} \int d^{2} p e^{i p[x-y)} \frac{p^{2}+m^{2}}{p^{2}+m^{2}} \exp \frac{-\left(p^{2}+m^{2}\right)}{\gamma^{2 N}} .
\end{aligned}
$$


In this way O.S. positivity is lost and at any fixed cutoff there is not a corresponding regularized Minkowski field, but the resulting euclidean measure is supersymmetric, meaning by that the expecation values with respect to this measure obey the Euclidean version of the Ward Identities associated to the formal supersymmetric theory (see remark at the end of this section and Appendix B) $\phi, \psi, F$.

b) It is well known that at $d=2$ the scalar massless field is not well defined. This can be cured in the Minkowski formalism (see Wightman [9] and references therein) defining $\phi_{M}, \psi_{M}, F_{M}$ as operator valued distributions over the test functions:

$$
\mathscr{S}_{0}=\{f \in \mathscr{S} \mid f(p=0)=0\} .
$$

This allows us to give a consistent $C^{*}$-algebraic definition of the observables of the theory. This procedure is equivalent to the introduction of the following regularization:

$$
\phi_{M}^{[\leq N]}(x)=\left(\phi_{M} * h^{[\leqq N]}\right)(x),
$$

where $h^{[\leqq N]}(p)=h_{0}(p) \exp \frac{-p^{2}}{2 \gamma^{2 N}}$ and $h_{0}(p) \rightarrow 0$ as $p_{1} \rightarrow 0$ so that

$$
\int d p_{1} \frac{h_{0}(p)}{p^{1}}<\infty .
$$

This extra regularization does not spoil the supersymmetry of the theory provided it is consistently introduced in the definition of all the three fields $\phi, \psi$, and $F$ although for the last two it will not be a priori needed.

We shall translate this procedure for our regularized Euclidean field. As (see next point c) we consider the finite volume case the previous condition to build a sensible theory amounts simply to eliminate the $p_{1}=0$ mode in the sum over $p_{1}$ which replaces the corresponding integral.

c) As we are studying the zero mass case we expect it to be really difficult to perform the infinite volume limit. Therefore we consider the finite volume theory and the choice of the boundary conditions is a delicate point. There are still two alternatives:

i) To consider a finite space volume.

ii) To consider a finite space-time volume.

In the first case we study the Schwinger functions of a finite space volume, say the interval $\left[\frac{-L}{2}, \frac{L}{2}\right]$. The existence and the analyticity of the Schwinger functions can be proven, as it will be discussed in the next sections, whatever boundary conditions are imposed, but to keep this finite volume model supersymmetric we have to impose periodic boundary conditions, in the space direction, to all three fields. 
The Euclidean theory is defined on space time cylinders and its covariances are

$$
\begin{gathered}
C^{\mathrm{L} \leqq N]}(x, y)=\frac{1}{L} \sum_{p_{1} \in \frac{2 \pi}{L} Z ; p_{1} \neq 0} \frac{1}{(2 \pi)} \int d p_{0} e^{i p(x-y)} \frac{1}{p_{1}^{2}+p_{0}^{2}} \exp \frac{-p^{2}}{\gamma^{2 N}}, \\
S^{[\leqq N]}(x, y)=\frac{1}{L} \sum_{p_{1} \in \frac{2 \pi}{L} Z ; p_{1} \neq 0} \frac{1}{(2 \pi)} \int d p_{0} e^{i p(x-y)} \frac{i p^{E}}{p_{0}^{2}+p_{1}^{2}} \exp \frac{-p^{2}}{\gamma^{2 N}}, \\
D^{[\leqq N]}(x, y)=\frac{1}{L} \sum_{p_{1} \in \frac{2 \pi}{L} Z ; p_{1} \neq 0} \frac{1}{(2 \pi)} \int d p_{0} e^{i p(x-y)} \exp \frac{-p^{2}}{\gamma^{2 N}} .
\end{gathered}
$$

In the second case, to preserve the supersymmetry, periodic boundary conditions are imposed both in space and time directions and the covariances associated to the free measure are obtained from the previous ones just substituting the integration over $p_{0}$ with the corresponding sum.

Notice that imposing periodic boundary conditions both in space and time directions, when we consider the finite space-time volume case, we are studying the Euclidean counterpart of the Supertrace (see Cecotti and Girardello [10]) instead of the finite temperature states which will require for the fermions antiperiodic boundary conditions in the time direction.

Remarks. We recall that the regularized fermionic measure is defined in the following way:

$$
\left.\left.\mu\left(d \psi^{[\leqq N]}\right)=\mathscr{N}_{\psi}^{-1} \int \mathscr{D} \psi^{[\leqq N]}\left[\exp \int d^{2} x \psi^{(2)[\leqq N]}\left(\gamma^{\mu} \partial_{\mu}\right)_{A} \psi^{(1)[\leqq N]}\right)\right)\right],
$$

where

and

$$
\mathscr{D} \psi^{[\leqq N]}=\prod_{q \in \frac{2 \pi}{L} Z^{2} ; q_{1}>0}\left[d \psi_{1}^{(1)}(q) d \psi_{2}^{(1)}(q) d \psi_{1}^{(1)}(-q) d \psi_{2}^{(1)}(-q)\right]
$$

$$
\left(\gamma^{\mu} \partial_{\mu}\right)_{\Lambda}^{[\leqq N]}=\left(S^{[\leqq N]}(x, y)\right)^{-1} .
$$

The regularized free measure given, the interacting one is obtained multiplying it by $e^{V[N N]}$, where $V^{[\leqq N]}$ is now expressed in terms of regularized fields. Therefore the regularized interacting measure of the theory is:

$$
\begin{aligned}
& \langle(\cdot)\rangle_{\Lambda}^{\leqq N]}=\mathcal{N}^{-1} \int P\left(d \phi^{[\leqq N]}\right) \mu\left(d \psi^{[\leqq N]}\right) P\left(d F^{[\leqq N]}\right) e^{\left.\left.V[\leqq N]\left(\phi^{[} \leqq N\right], \psi^{[} \leqq N\right], F[\leqq N]\right)}(\cdot), \\
& V^{[\leqq N]}\left(\phi^{[\leqq N]}, \psi^{[\leqq N]}, F^{[\leqq N]}\right)=\frac{\lambda}{2} \int d^{2} x\left[\psi^{(2)^{[\leqq N]}} \psi^{(1)^{[\leqq N]}} a^{2} \cos \alpha \phi^{[\leqq N]}\right. \\
& \left.+2 i F^{[\leqq N]} \alpha \sin \alpha \phi^{[\leqq N]}\right] \text {, }
\end{aligned}
$$

and we can also integrate with respect to the auxiliary field $F^{[\leqq N]}$ obtaining

$$
\begin{aligned}
\left.\langle(\cdot)\rangle_{A} \leqq N\right] & =\mathcal{N}^{-1} \int P\left(d \phi^{[\leqq N]}\right) \mu\left(d \psi^{[\leqq N]}\right) e^{V^{[\leqq N]}\left(\phi^{[\leqq N]}, \psi^{[\leqq N]}\right)}(\cdot) \\
V^{[\leqq N]}\left(\phi^{[\leqq N]}, \psi^{[\leqq N]}\right)= & \frac{1}{2} \lambda_{B} \alpha^{2} \int_{\Lambda} d^{2} x\left(\psi^{(2)^{[\leqq N]}}(x) \psi^{(1)^{[\leqq N]}}(x)\right) \cos \alpha \phi^{[\leqq N]}(x) \\
& +\frac{-1}{2} \lambda_{B}^{2} \alpha^{2} \int_{\Lambda x \Lambda} d^{2} x d^{2} y \sin \alpha \phi^{[\leqq N]}(x) \sin \alpha \phi^{[\leqq N]}(y) D^{[\leqq N]}(x, y)
\end{aligned}
$$


where we call $\lambda: \lambda_{B}$ (see the next section) and with $\Lambda$ we indicate the finite space time: $\left[\frac{-L}{2}, \frac{L}{2}\right] \times\left[\frac{-L}{2}, \frac{L}{2}\right]$ or the cylinder: $(-\infty, \infty) \times\left[\frac{-L}{2}, \frac{L}{2}\right]$.

Remarks. i) The supersymmetry of the interacting measure is a direct consequence of the previous definitions. In fact the action can be expressed in terms of the regularized superfield and is therefore invariant under the transformations of the fields $\phi^{[\leqq N]}, \psi^{[\leqq N]}, F^{[\leqq N]}$ which are exactly of the same kind as those of the fields $\phi$, $\psi, F$. The variation $\delta \phi^{[\leqq N]}$, for instance, is obtained from the formal expression $\delta \phi=Q \Phi$ just substituting everywhere the regularized fields to the non-regularized ones and performing the other substitutions listed in Eqs. (2.1) and (2.2) (see Appendix B).

ii) With our definitions it follows immediately that after integrating over the auxiliary field $F^{[\leqq N]}$ a non-local part appears in the regularized Hamiltonian and therefore in the potential $V^{[\leqq N]}$ of $(2.21)$.

The non-local character of this contribution to the interaction is a consequence of our symmetry preserving way of regularizing the model and it will disappear in the limit $N \rightarrow \infty$.

iii) If we consider a regularized Minkowski theory it is easy to convince oneself that, after the elimination of the zero mode, the field commutators differ from the canonical ones by an extra term that goes to zero in the infinite volume limit. This non-local feature is present also in our model and does not disappear in the limit $N \rightarrow \infty$.

iv) The regularized covariances proposed in Eqs. (2.15), although perfectly appropriate in the massive case turn out not to have all the right properties in the massless case especially when we want to decompose them in a sum of covariances associated to fields of definite frequency following the well known philosophy of the renormalization group.

This will be discussed in detail in Sect. 4.

\section{The Introduction of the Wick Product}

Generally in Supersymmetric theories one is not allowed to introduce the Wick product as this would spoil the supersymmetry of the theory or its introduction would be useless as the extra bosonic terms will compensate with the extra fermionic ones. The situation here is different because the supersymmetric SineGordon theory is not a polynomial theory and to consider : $\cos \alpha \phi(x)$ : instead of $\cos \alpha \phi(x)$ means to multiply it by a (infinite) constant:

$$
: \cos \alpha \phi(x):=\exp \frac{\alpha^{2}}{2}\left\langle\phi(x)^{2}\right\rangle \cos \alpha \phi(x) .
$$

On the other side it is known, (see Sect. 9), that the scalar Sine-Gordon theory in a finite volume is trivial, when $\alpha^{2}<4 \pi$, if we do not introduce the Wick product. This is what we do here also in the following way: we impose that the bare coupling constant $\lambda_{B}$ we have introduced in (2.28) has the following $N$-dependence

$$
\lambda_{B}(N)=\lambda \exp \frac{\alpha^{2}}{2} C_{\Lambda}^{[\leqq N]}(0)=\lambda \gamma^{\left(\alpha^{2} / 4 \pi\right) N} C(L, \alpha),
$$


where $C(L, \alpha)$ is independent from $N$.

With this definition we have

$$
\begin{aligned}
V^{[\leqq N]}\left(\phi^{[\leqq N]}, \psi^{[\leqq N]}\right)= & \frac{1}{2} \lambda_{B} \alpha^{2} \int_{\Lambda} d^{2} x\left(\psi^{(2)[N]}(x) \psi^{(1)^{[\leqq N]}}(x)\right): \cos \alpha \phi^{[\leqq N]}(x): \\
& \left.-\frac{1}{2} \lambda_{B}^{2} \alpha^{2} \int_{\Lambda \times \Lambda} d^{2} x d^{2} y: \sin \alpha \phi^{[\leqq N]}(x):: \sin \alpha \phi^{[\leqq N](} y\right): D^{[\leqq N]}(x, y) .
\end{aligned}
$$

\section{The Effective Potential}

The construction of the theory in the space time volume $\Lambda$ in the limit of the ultraviolet cutoff going to infinity will be done constructing the effective potential of the theory for any length scale $\gamma^{k}\left(k \in Z^{+}\right)$(see for instance Gallavotti et al. [4], Gawedzki and Kupiainen [12]). The techniques used to build it will be those of the "Renormalization Group." The effective potential with a finite cutoff $N, V_{N}^{(k)}$ (we omit the volume dependence) is defined by

$$
e^{V_{N}^{(k)}}=\int \prod_{k+1}^{N} P\left(d \phi^{(h)}\right) \prod_{k+1}^{N} \mu\left(d \psi^{(h)}\right) e^{V^{(N)}},
$$

where we have alredy eliminated the auxiliary field by integrating it out. $P\left(d \phi^{(h)}\right)$ and $\mu\left(d \psi^{(h)}\right)$ are defined by the following equations:

$$
P\left(d \phi^{[\leqq N]}\right)=\prod_{0}^{N} P\left(d \phi^{(h)}\right), \quad \mu\left(d \psi^{[\leqq N]}\right)=\prod_{0}^{N} h\left(d \psi^{(h)}\right) .
$$

Following the last remark of Sect. 2, the fields $\phi^{[\leqq N]}, \psi^{[\leqq N]}$, and $F^{[\leqq N]}$ have the following covariances, different from those introduced in Eqs. (2.15):

$$
\begin{gathered}
C^{[\leqq N]}(x, y)=\frac{1}{L} \sum_{p_{1} \in \frac{2 \pi}{L} Z ; p_{1} \neq 0} \frac{1}{(2 \pi)} \int d p_{0} e^{i p(x-y)} \frac{1}{p_{1}^{2}+p_{0}^{2}} \exp \frac{-p^{2}}{\gamma^{2 N}} \exp \frac{-p_{1}^{2}}{\gamma^{2 N}}, \\
S^{[\leqq N]}(x, y)=\frac{1}{L} \sum_{p_{1} \in \frac{2 \pi}{L} Z ; p_{1} \neq 0} \frac{1}{(2 \pi)} \int d p_{0} e^{i p(x-y)} \frac{i \not p}{p_{0}^{2}+p_{1}^{2}} \exp \frac{-p^{2}}{\gamma^{2 N}} \exp \frac{-p_{1}^{2}}{\gamma^{2 N}} \\
D^{[\leqq N]}(x, y)=\frac{1}{L} \sum_{p_{1} \in \frac{2 \pi}{L} Z ; p_{1} \neq 0} \frac{1}{(2 \pi)} \int d p_{0} e^{i p(x-y)} \exp \frac{-p^{2}}{\gamma^{2 N}} \exp \frac{-p_{1}^{2}}{\gamma^{2 N}}
\end{gathered}
$$

Then we perform the following decomposition:

$$
\begin{aligned}
C^{\mathrm{L} \leqq N]}(x, y)= & \sum_{h=0}^{N} \sum_{l=0}^{N} C^{(h, l)}(x, y)=\sum_{h=0}^{N} \sum_{l=0}^{N} \int d p_{0} e^{i p(x-y)} \frac{1}{p_{1}^{2}+p_{0}^{2}} \\
& \times\left(\exp \frac{-p^{2}}{\gamma^{2 h}}-\exp \frac{-p^{2}}{\gamma^{2(h-1)}}\right)\left(\exp \frac{-p_{1}^{2}}{\gamma^{2 l}}-\exp \frac{-p_{1}^{2}}{\gamma^{2(l-1)}}\right)
\end{aligned}
$$

with the convention that

$$
\exp \frac{-p^{2}}{\gamma^{-2}}=0, \quad \exp \frac{-p_{1}^{2}}{\gamma^{-2}}=0 .
$$


Finally we make this partial resummation of the covariances:

$C^{[\leqq N]}(x, y)=\sum_{h=0}^{N}\left(\sum_{l=h+1}^{N} C^{(l, h)}(x, y)\right)+\sum_{h=0}^{N}\left(\sum_{l=h}^{N} C^{(h, l)}(x, y)\right):=\sum_{h=0}^{N} \hat{C}^{(h)}(x, y)$.

The same kind of decomposition has to be performed for $S^{[\leqq N]} . P\left(d \phi^{(h)}\right)$, and $\mu\left(d \psi^{(h)}\right)$ have the same structure as $P\left(d \phi^{[\leqq N]}\right)$ and $\mu\left(d \psi^{[\leqq N]}\right)$ with $C^{[\leqq N]}$ and $S^{[\leqq N]}$ substituted by $\widehat{C}^{(h)}$ and $\widehat{S}^{(h)}$.

Remark. The reason for such an involved way to regularize the fields is that with the standard regularization proposed in (2.15), when the mode $p_{1}=0$ is eliminated, the covariances $C^{(h)}$ and $S^{(h)}$ corresponding to the regularization $\left(\exp \frac{-p^{2}}{\gamma^{2 h}}-\exp \frac{-p^{2}}{\gamma^{2(h-1)}}\right)$ do not have the right decay properties. It is a long but simple task to show that, vice versa the needed exponential decay properties on the right scale are shared by the covariances $\hat{C}^{(h)}$ and $\hat{S}^{(h)}$.

The Gaussian independent fields $\phi^{(h)}$ are associated to the $\hat{C}^{(h)}$ 's and the Grassmann fields $\psi^{(h)}$ to the $\hat{S}^{(h)}$ 's. The following relations follow:

$$
\phi^{[\leqq N]}=\sum_{0}^{N} h \phi^{(h)}, \quad \psi^{[\leqq N]}=\sum_{0}^{N} h \psi^{(h)} .
$$

The existence of the theory is defined as the existence of the limit

$$
\lim _{N \rightarrow \infty} \frac{V_{N}^{(k)}\left(\phi^{[\leqq k]}, \psi^{[\leqq k]}\right)}{|\Lambda|}:=\frac{V^{(k)}\left(\phi^{[\leqq k]}, \psi^{[\leqq k]}\right)}{|\Lambda|},
$$

where, if $\Lambda$ is a space-timer cylinder, dividing by $|\Lambda|$ means to divide by the volume of a truncated cylinder and then performing the limit toward the infinite cylinder.

Remark. Here and in the sequel we use the same notation $V_{N}^{(k)}$ for $V(\phi, \psi)$ and for $V(\phi, \psi, F)$, but no ambiguity can arise in any specific context.

\section{The Results: The Existence and the Analyticity of the Theory}

The proof of the existence of the theory is achieved by proving that the effective potential $V_{N}^{(k)}$ admits a well defined limit as $N$ goes to infinity, for a fixed volume. This result, although hard to obtain, is, nevertheless, someway expected as, for $\alpha^{2}<4 \pi$, the theory is superrenormalizable (compared to the scalar case, (see Sect. 9), the properties of this model are similar if we shift $\alpha^{2}$ by $4 \pi$ ).

What is more relevant is that for $\alpha^{2}<2 \pi$ one can extend to the supersymmetric model another result proved by Fröhlich and Seiler [13], for $\alpha^{2}<4 \pi$, and then with a different technique by Benfatto [14] when $\alpha^{2}<6 \pi$, namely that the theory is analytic. Analyticity means that the effective potential can be written as a power series in the coupling constant $\lambda$, each term being a functional of the fields $\phi$ and $\psi$ such that its coefficient satisfies an appropriate bound. This allows us immediately to obtain the Schwinger functions of the theory or its vacuum energy as a convergent power series in $\lambda$ in a appropriate disk around $\lambda=0$ of the complex $\lambda$ plane. 
We stress that this result is independent on which boundary conditions we choose. The choice of the boundary conditions is crucial only for the results described in Corollary 3.

We state, now, a theorem which collects the results already discussed.

Theorem 1. Let $V^{(N)}=V_{\Lambda}^{[\leqq N]}\left(\phi^{[\leqq N]}, \psi^{[\leqq N]}\right)$ if we impose periodic b.c. ( see Eq. (3.3)),

$$
V^{(N)}=V_{\Lambda}^{[\leqq N]}\left(\phi^{[\leqq N]}, \psi^{[\leqq N]}\right)+\sum_{0}^{M(\alpha)} C_{k}(L, \alpha, N) \lambda^{k},
$$

otherwise, where the $C_{k}(L, \alpha, N)$ are constant counterterms uniformly bounded in $N$. Let $V_{N}^{(k)}$ be the effective potential defined in (4.1) where the covariances associated to the free measures have the appropriate boundary conditions.

Then: i) The effective potential $V_{N}^{(k)}$ has the following expression:

$$
\begin{aligned}
V_{N}^{(k)}= & \sum_{1}^{\infty} \lambda^{n} \sum_{\sigma_{1} \ldots \sigma_{n}}^{(-1,+1)} \sum_{\beta_{1} \ldots \beta_{2 n}}^{(0,1)} \int_{\Lambda^{n}} d x^{1} \ldots d x^{n} W_{n ; N}^{(k)}(\mathbf{x}, \boldsymbol{\sigma}, \boldsymbol{\beta})\left[: e^{i \alpha \sum_{1}^{n} \cdot \sigma_{i} \phi[\leqq k]\left(x_{i}\right)}:\right. \\
& \left.\times \psi_{1}^{[\leqq k]}\left(x_{1}\right)^{\beta_{1}} \psi_{2}^{[\leqq k]}\left(x_{1}\right)^{\beta_{2}} \ldots \psi_{1}^{[\leqq k]}\left(x_{n}\right)^{\beta_{2 n-1}} \psi_{2}^{[\leqq k]}\left(x_{n}\right)^{\beta_{2 n}}\right] \\
& +(\text { constant terms })
\end{aligned}
$$

(here all the $\psi$ 's are $\psi^{(1)} s$ ).

ii) We define the following norm

$$
\left\|W_{n ; N}^{(k)}\right\|=\sum_{\sigma} \cdot \sum_{\beta} \cdot \int_{\Lambda^{n}} \mathbf{d x} \delta\left(x_{1}\right)\left|W_{n ; N}^{(k)}(\mathbf{x}, \boldsymbol{\sigma}, \boldsymbol{\beta})\right|,
$$

then, if $\alpha^{2}<2 \pi$ we have the following inequalities:

$$
\left\|W_{n ; N}^{(k)}\right\| \leqq C^{n}, \quad k \geqq 0, \quad \forall n \in Z^{+},
$$

where $C$ depends on $\alpha$ but is $k, N, L$ independent,

$$
\left\|W_{n ; N}^{(k=-1)}\right\| \leqq(C(L))^{n},
$$

where $C(L)$ is still $N$ independent, but $\lim _{L \rightarrow \infty} C(L)=\infty$.

From Theorem 1 easily follows:

Corollary 1. Let us define

$$
V_{N}^{(-1)}:=\log \int P\left(d \phi^{[\leqq N]}\right) \mu\left(d \psi^{[\leqq N]}\right) e^{\left.\left.V[\leqq N]\left(\phi^{[} \leqq N\right], \psi^{[} \leqq N\right]\right)} .
$$

Then, if $\alpha^{2}<2 \pi, V_{N}^{(-1)}$ is, for any $N$ and also in the $N \rightarrow \infty$ limit, at fixed $\Lambda$, an analytic function of $\lambda$ in a disk of the $\lambda$ complex plane whose radius depends on $L$ but not on $N$ and shrinks to zero as $L \rightarrow \infty$.

Proof. Define $\phi^{(-1)}=\psi^{(-1)}=0$ and substitute -1 in the place of $k$ in the previous theorem.

Remark. From this theorem the expectation values of the theory at finite spacevolume are analytic functions of $\lambda$. Since our estimates of the convergence radius of the series in $\lambda$ shrinks to zero in the infinite volume limit we expect that nonperturbative effects could arise in this case. 
The proof of Theorem 1 is rather cumbersome and its details will be given in the companion paper (II). In Sect. 8 of this paper we briefly discuss the strategy of the proof and the techniques used.

Looking through the proof it will be easily recognized that the choice of the boundary conditions is not relevant and this is expected since this result is the solution of a "ultraviolet" problem where we never use the supersymmetry of the theory. A complete different situation, vice versa, is that of the next result where to preserve the supersymmetry after the regularization turns out to be crucial.

Corollary 2. For arbitrary $N$ and $L$ and periodic boundary conditions:

$$
V_{N}^{(-1)}=0,
$$

both in the infinite cylinder and in the finite space-time volume case,

Proof. By definition

$$
\int P\left(d \phi^{[\leqq N]}\right) \mu\left(d \psi^{[\leqq N]}\right) P\left(d F^{[\leqq N]}\right) e^{V[\leqq N]}:=e^{V[-1]},
$$

and since, by Corollary 1 , we have that $V_{N}^{(-1)}$ is an analytic function of $\lambda$, recalling Eq. (2.26) we have that

$$
\begin{aligned}
& \frac{\partial}{\partial \lambda} \log \int_{\text {p.b.c. }} P\left(d \phi^{[\leqq N]}\right) \mu\left(d \psi^{[\leqq N]}\right) P\left(d F^{[\leqq N]}\right) e^{V[N N]} \\
& =\frac{1}{2} \int_{\Lambda} d^{2} x\left\langle\psi^{(2) ! \leqq N]}(x) \psi^{(1) ! \leqq N]}(x) \alpha: \cos \alpha \phi^{[\leqq N]}(x):\right. \\
& \left.\quad+2 i F^{[\leqq N]}: \sin \alpha \phi^{[\leqq N]}(x):\right\rangle_{\lambda, \Lambda, N},
\end{aligned}
$$

where

$$
\langle(\cdot)\rangle_{\lambda, \Lambda, N}:=\mathscr{N}^{-1} \int_{\text {p.b.c. }} P\left(d \phi^{[\leqq N]}\right) \mu\left(d \psi^{[\leqq N]}\right) P\left(d F^{[\leqq N]}\right) e^{V I \leqq N]}(\cdot) .
$$

As, see Eqs. (2.6), (2.7):

$$
\left(\psi^{(2)} \cdot \psi^{(1)}\right)=\sum_{\alpha} \psi_{\alpha}^{(2)} \psi_{\alpha}^{(1)}=-2 i \psi_{1}^{(1)} \psi_{2}^{(1)}=-2 i \psi_{1} \psi_{2},
$$

and in Appendix B, see Eq. (B.9), the following relation is proven:

$$
\alpha\left\langle\psi_{1}^{[\leqq N]}(x) \psi_{2}^{[\leqq N]}(x) \cos \alpha \phi^{[\leqq N]}(x)\right\rangle_{\lambda, \Lambda, N}=\left\langle F^{[\leqq N]}(x) \sin \alpha \phi^{[\leqq N]}(x)\right\rangle_{\lambda, \Lambda, N} .
$$

It follows that $\frac{\partial}{\partial \lambda} V_{N}^{(-1)}(\lambda)=0$ for any $N$ and $\lambda$ such that $|\lambda|<\lambda_{0}(L)$ and since $V_{N}^{(-1)}(0)=0$ Corollary 2 holds.

\section{The Lattice Regularization and O.S. Positivity}

In this section we will study a discrete version of the model associated to the formal action (1.14). We will discuss explicitly only the finite space-time volume case, but the same results hold in the infinite-cylinder case.

Suppose that the region $\Lambda$ is a square of side $L$ (integer) and consider a square lattice $Z_{a}$, with spacing $a$, such that $\frac{L}{2 a}$ is an integer. If we define on the lattice $Z_{a}$ a 
scalar field $\phi(\underline{n} a)$ and two Maiorana spinor fields $\psi^{(\kappa)}(\underline{n} a),(\kappa=1,2)$ we can write the following Euclidean action:

$$
\begin{aligned}
\mathscr{A}= & \frac{1}{2} \sum_{\underline{n} \in \Lambda} \sum_{\mu=1 ; 2}\left(\phi\left(\underline{n} a+\underline{e}_{\mu} a\right)-\phi(\underline{n} a)\right)^{2} \\
& +\frac{a}{2} \sum_{n \in \Lambda} \sum_{\mu=1 ; 2}\left[\psi^{(2)}(\underline{n} a)\left(-\gamma^{\mu E}\right) \psi^{(1)}\left(\underline{n} a+\underline{e}_{\mu} a\right)\right. \\
& \left.-\psi^{(2)}\left(\underline{n} a+\underline{e}_{\mu} a\right)\left(-\gamma^{\mu E}\right) \psi^{(1)}(\underline{n} a)\right] \\
& +a^{2} \sum_{n \in \Lambda}\left(-\psi^{(2)}(\underline{n} a) \psi^{(1)}(\underline{n} a) \lambda \alpha^{2} \cos \alpha \phi(\underline{n} a)\right. \\
& -\sum_{n \in \Lambda} \sum_{n^{\prime} \in \Lambda} \sin \alpha \phi(\underline{n} a) \sin \alpha \phi\left(\underline{n}^{\prime} a\right) D^{a}\left(\underline{n} a-\underline{n}^{\prime} a\right),
\end{aligned}
$$

where $\underline{e}_{\mu}$ are the lattice unit vectors and

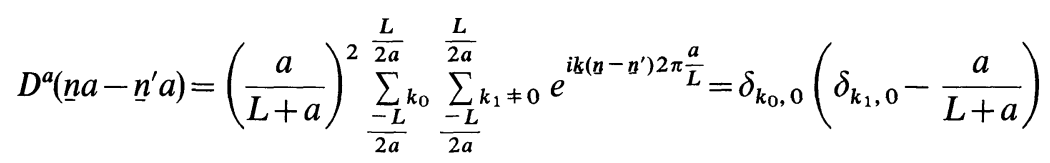

is the discrete version of the $D^{[\leqq N]}(x, y)$ introduced in Eq. (2.15).

Remark. Often, in the literature, another term is added to the fermionic part of the action to avoid the "proliferation" of the fermions [15]. This term is not needed here because we will consider the limit $a \rightarrow 0$ but its presence would not affect the forthcoming arguments. Starting from the action (6.1) we obtain the following measure:

$$
\frac{1}{\mathscr{N}} P_{a}(d \phi) \mu_{a}(d \psi) e^{V_{a}(\phi, \psi)}
$$

where

$$
\begin{aligned}
V_{a}(\phi, \psi)= & a^{2} \sum_{n \in \Lambda}\left(-\psi^{(2)}(\underline{n} a) \psi^{(1)}(\underline{n} a) \lambda \alpha^{2} \cos \alpha \phi(\underline{n} a)\right) \\
& -a^{2} \sum_{n \in \Lambda} \sum_{n^{\prime} \in \Lambda} \sin \alpha \phi(\underline{n} a) \sin \alpha \phi\left(\underline{n}^{\prime} a\right) D^{a}\left(\underline{n} a-\underline{n}^{\prime} a\right)
\end{aligned}
$$

and $P_{a}(d \phi), \mu_{a}(d \psi)$ are defined by their covariances:

$$
\begin{aligned}
& C_{a}\left(\underline{n} a-\underline{n}^{\prime} a\right)=\left(\frac{a}{L+a}\right)^{2} \sum_{\frac{-L}{2 a}}^{\frac{L}{2 a}} k_{0} \sum_{\frac{-L}{2 a}}^{\frac{L}{2 a}} k_{1} \neq 0 \frac{e^{i k\left(n-n^{\prime}\right) 2 \pi \frac{a}{L}}}{2 \sum_{0}^{1}\left(1-\cos 2 \pi \frac{a}{L} k_{\mu}\right)}, \\
& S^{a}\left(\underline{n} a-\underline{n}^{\prime} a\right)=\left(\frac{a}{(L+a)^{2}}\right) \sum_{\frac{-L}{2 a}}^{\frac{L}{2 a}} k_{0} \sum_{\frac{-L}{2 a}}^{\frac{L}{2 a}} k_{1} \neq 0 \frac{e^{i k\left(n-n^{\prime}\right) 2 \pi \frac{a}{L}}}{2 \sum_{0} \mu\left(\gamma^{\mu} \sin 2 \pi \frac{a}{L} k_{\mu}\right)} .
\end{aligned}
$$

For any finite $a$ and $L$ the measure (6.3) is well defined and it is a standard matter to check that it is also O.S. positive. 
To study the limit $a \rightarrow 0$ we introduce in analogy with the continuous case [see Eqs. (4.5) and (4.6)] the independent random fields associated to well defined "frequencies" $\phi^{(h)}$ and $\psi^{(h)}$ such that:

$$
\phi(\underline{n} a)=\sum_{0}^{N} h \phi^{(h)}(\underline{n} a), \quad \psi(\underline{n} a)=\sum_{0}^{N} h \psi^{(h)}(\underline{n} a) .
$$

The fields $\phi^{(h)}$ and $\psi^{(h)}$ are defined through a decomposition of the covariances $C^{a}\left(\underline{n} a-\underline{n}^{\prime} a\right)$ and $S^{a}\left(\underline{n} a-\underline{n}^{\prime} a\right)$ of the same kind as that described in Sect. 4, Eqs. (4.4), (4.5).

We denote $2 \sum_{0}^{1}\left(1-\cos 2 \pi \frac{a}{L} k_{\mu}\right):=p^{2} a^{2}$ and we rewrite the denominator of $C^{a}\left(\underline{n} a-\underline{n}^{\prime} a\right)$ in the following way:

$$
\frac{1}{p^{2}}=\frac{p_{1}^{2}}{p^{2} p_{1}^{2}}=p_{1}^{2} \sum_{h=0}^{N} \sum_{l=0}^{N} G\left(p^{2} ; h, l\right)
$$

with

$$
\begin{gathered}
G\left(p^{2} ; h, l\right)=\left(\frac{1}{p^{2}+\gamma^{2(h-1)}}-\frac{1}{p^{2}+\gamma^{2 h}}\right)\left(\frac{1}{p_{1}^{2}+\gamma^{2(l-1)}}-\frac{1}{p_{1}^{2}+\gamma^{2 l}}\right) \quad h, l>0, \\
G\left(p^{2} ; 0,1\right)=\left(\frac{1}{p^{2}}-\frac{1}{p^{2}+1}\right)\left(\frac{1}{p_{1}^{2}+\gamma^{2(l-1)}}-\frac{1}{p_{1}^{2}+\gamma^{2 l}}\right) \\
G\left(p^{2} ; h, 0\right)=\left(\frac{1}{p^{2}+\gamma^{2(h-1)}}-\frac{1}{p^{2}+\gamma^{2 h}}\right)\left(\frac{1}{p_{1}^{2}}-\frac{1}{p_{1}^{2}+1}\right) \\
G\left(p^{2} ; 0,0\right)=\left(\frac{1}{p^{2}}-\frac{1}{p^{2}+1}\right)\left(\frac{1}{p_{1}^{2}}-\frac{1}{p_{1}^{2}+1}\right) .
\end{gathered}
$$

In the last three terms the fact that $p_{1}$ is different from zero is crucial. Therefore

$$
C^{a}\left(\underline{n} a-\underline{n}^{\prime} a\right)=\sum_{n=0}^{N} \sum_{l=0}^{N} C^{(h, l)}\left(\underline{n} a-\underline{n^{\prime}} a\right),
$$

where $N$ is defined by the relation: $a=\gamma^{N}$.

The same decomposition is applied to $S^{a}\left(\underline{n} a-\underline{n}^{\prime} a\right)$ after we rewrite its denominator as

$$
\frac{1}{\sum_{0}^{1}\left(\gamma^{\mu} \sin 2 \pi \frac{a}{L} k_{\mu}\right)}=\frac{\sum_{0}^{1}\left(\gamma^{\mu} \sin 2 \pi \frac{a}{L} k_{\mu}\right)}{p^{2}}=\sum_{0}^{1}\left(\gamma^{\mu} \sin 2 \pi \frac{a}{L} k_{\mu}\right) \frac{p_{1}^{2}}{p^{2} p_{1}^{2}} \text {. }
$$

We proceed now exactly as in Sect. 4 and we define the covariances $\hat{C}^{(h)}\left(\underline{n} a-\underline{n}^{\prime} a\right)$ and $\widehat{S}^{(h)}\left(\underline{n} a-\underline{n}^{\prime} a\right)$ through the same relations as before:

$$
\begin{aligned}
C\left(\underline{n} a-\underline{n}^{\prime} a\right) & =\sum_{h=0}^{N}\left(\sum_{l=h+1}^{N} C^{(l, h)}\left(\underline{n} a-\underline{n}^{\prime} a\right)\right)+\sum_{h=0}^{N}\left(\sum_{l=h}^{N} C^{(h, l)}\left(\underline{n} a-\underline{n}^{\prime} a\right)\right) \\
& :=\sum_{h=0}^{N} \hat{C}^{(h)}\left(\underline{n} a-\underline{n}^{\prime} a\right)
\end{aligned}
$$

and the analogous one for $S\left(\underline{n} a-\underline{n}^{\prime} a\right)$. 
$\hat{C}^{(h)}\left(\underline{n} a-\underline{n}^{\prime} a\right)$ and $\hat{S}^{(h)}\left(\underline{n} a-\underline{n}^{\prime} a\right)$ are the covariances associated to the fields $\phi^{(h)}$ and $\psi^{(h)}$. As in the continuous case it is a standard task to show that these covariances have the exponential decay on the right scale, namely $\hat{C}^{(h)}\left(\underline{n} a-\underline{n}^{\prime} a\right)$ decays exponentially on the scale $\gamma^{-h}$.

The general properties of the covariances $\hat{C}^{(h)}\left(\underline{n} a-\underline{n}^{\prime} a\right)$ and $\hat{S}^{(h)}\left(\underline{n} a-\underline{n}^{\prime} a\right)$ allow us to prove, also in the lattice case, Theorem 1 and Corollaries 1 and 2 of Sect. 5.

Therefore, as the limit $a \rightarrow 0$ of the lattice theory is the same as the limit $N \rightarrow \infty$ of the continuous theory (each term of the perturbative expansion is the same and in both cases the series are convergent) our limit Euclidean Theory is Supersymmetric and fulfills all the requirements of the O.S. reconstruction program.

\section{The Results: The Supersymmetry is not Spontaneously Broken}

The results of the previous two sections allow us to conclude that in this theory the supersymmetry is not spontaneously broken. In fact the possibility of going back to the Minkowski theory, via the Reconstruction theorem, allows to define field operators and charges also in the case of the limit theory and therefore to interpret the result of Corollary 2 as the fact that the Witten index of the theory is equal to one which just excludes the spontaneous breaking of the supersymmetry.

On the other side, as a corollary of the fact that the theory is analytic, it follows that the Euclidean Ward identities associated to the regularized theory are satisfied also by the limiting theory. This is enough to prove that the supersymmetry is not spontaneously broken without using explicitly Corollary 2.

Finally we remark that, as already pointed in subsection $b$ of Sect. 2, to define this massless theory, we are obliged to eliminate the zero mode from the beginning which, of course, makes in some sense obvious the fact that the resulting theory is not spontaneously broken.

\section{The Strategy of the Proofs}

From the discussion in Sects. 5 and 6 it is clear that all our results are based on the proof of Theorem 1. Roughly all amounts to have a well organized way of expanding the effective potential in a sum of terms we can separately bound with estimates good enough not to destroy the inequality (5.3).

This is obtained using the ideas of the Renormalization Group, performing one after the other the integrations over the different momentum scales (different "frequencies") so to use at its best the "locality" one has at each scale. The integration, frequency by frequency, coupled to the expansion in powers of $\lambda$ gives the so-called "tree expansion" that Gallavotti and one of the present authors(F.N.) have introduced in [4] to study the connection between the perturbative renormalization and the constructive one in the $\phi_{4}^{4}$ field theory. The expansion is such that to each tree (for a precise definition of a tree see [4]) is associated a well defined expression obtained combining many truncated expectations of products of fields on different scales. These terms can be well estimated and moreover at each order $\lambda^{n}$ their number grows as $C^{n}$ so that, if one is able to prove that no factorials are produced in the various estimates, one has the possibility of proving the analyticity of the theory. The details of the proof are complicated, also because 
one has to disentangle the bosonic and the fermionic factors to apply separately different estimates. The paper (II) is mainly devoted to the proof of Theorem 1.

\section{Some Remarks on the Scalar Sine-Gordon Field Theory}

We briefly recall some results on the scalar Sine-Gordon theory which have in part guided us in this investigation. It has been proven (Fröhlich et al. [13] and references therein) that if $\alpha^{2}<4 \pi$ the massive Sine-Gordon theory exists. The interaction for this theory is (its regularized version), in a finite volume,

$$
V_{A}^{[\leqq N]}=\lambda \int_{A} d^{2} x: \cos \alpha \phi^{[\leqq N]}(x):
$$

If $\alpha^{2}<4 \pi$, Fröhlich has proven, among other things, the ultraviolet stability:

$$
e^{E_{-}(\lambda)|\Lambda|} \leqq \int P_{\Lambda}\left(d \phi^{[\leqq N]}\right) e^{V_{\Lambda}^{[\leqq N]}} \leqq e^{E_{+}(\lambda)|\Lambda|},
$$

where $E_{(\cdot)}(\lambda)$ does not depend on $N$ and is $O(\lambda)$. If $\alpha^{2} \geqq 4 \pi$, Benfatto, Gallavotti and Nicolò, Nicolò and Nicolò, Renn and Steinmann [16] have proven that:

If

$$
\alpha^{2} \in\left[4 \pi, \alpha_{n}^{2}\right], \quad \alpha_{n}^{2}=8 \pi\left(1-\frac{1}{2 n}\right),
$$

the ultraviolet stability becomes

$$
\begin{aligned}
& \exp \left(\sum_{1}^{M(\alpha)} C_{k}(\Lambda, \alpha, N) \lambda^{k}+E_{-}(\lambda)|\Lambda|\right) \leqq \int P_{\Lambda}\left(d \phi^{[\leqq N]}\right) e^{V[\leqq N]} \\
& \quad \leqq \exp \left(\sum_{1}^{M(\alpha)} C_{k}(\Lambda, \alpha, N) \lambda^{k}+E_{+}(\lambda)|\Lambda|\right)
\end{aligned}
$$

where $C_{k}(\Lambda, \alpha, N)$ are constants diverging, if $k$ is even, as $N$ goes to infinity and $E_{(\cdot)}(\lambda)$ is $N$ independent and $O\left(\lambda^{n+\varepsilon}\right), \varepsilon>0$. From this result it follows that the Schwinger functions of the theory exist as any expression

$$
\frac{\int P_{\Lambda}\left(\phi^{[\leqq N]}\right) e^{V\left[I_{\Lambda} \leqq N\right]} \prod_{j} \phi^{[\leqq N]}\left(f_{j}\right)}{\int P_{\Lambda}\left(d \phi^{[\leqq N]}\right) e^{\left.V_{\Lambda} \leq N\right]}}
$$

is equal to the same expression with $V_{A}^{[\leqq N]}$ replaced by $V_{A}^{[\leqq N]}-\sum_{1}^{M(\alpha)} C_{k}(\Lambda, \alpha, N) \lambda^{k}$ which satisfies the ultraviolet stability.

The three following remarks conclude and justify this very short summary.

$\alpha$ ) It is clear that as $\alpha^{2} \rightarrow 8 \pi$ the number of counterterms tend to infinity; at $\alpha^{2}=8 \pi$ the theory needs also field dependent counterterms and it is not anymore superrenormalizable but only renormalizable. Above $8 \pi$ the theory is not renormalizable. We expect that a similar situation occurs in the Supersymmetric model at $\alpha^{2}=4 \pi$.

$\beta)$ To insert the Wick product is equivalent to have a theory without the Wick product but with interaction

$$
\lambda \exp \left(\frac{\alpha^{2}}{2} C_{\Lambda}^{[\leqq N]}(0)\right) \int_{A} d^{2} x \cos \alpha \phi^{[\leqq N]}(x),
$$


with the coupling constant going to infinity when $N \rightarrow \infty$ as $\lambda \gamma^{\left(\alpha^{2} / 4 \pi\right) N}\left(\gamma>1, \gamma^{N}\right.$ is the ultraviolet cutoff).

$\gamma$ ) If $\alpha^{2}<6 \pi$, Benfatto [14] has proven that the theory is analytic in the sense previously discussed for the supersymmetric theory.

\section{Conclusions and Discussions}

It is now time to complete the discussion about the meaning of the two dimensional massless theory. If the masses are zero, as we have already discussed at the end of Sect. 2, we have to subtract the zero mode to get a well defined theory (see Wigthman [9]). In the finite volume case the resulting theory is not local; this is reflected by the fact that, eliminating the zero mode, the free fields do not satisfy the usual commutation relations for the presence of a constant term which goes to zero as the volume tends to infinity. The presence of the interaction does not cure, at least at finite volume, this problem.

Nevertheless the Euclidean regularized theory is still supersymmetric in the sense that the Ward identities are satisfied. As it is possible to prove that in the limit of removing the cutoff the Hamiltonian and the charges can be built, using the reconstruction theorem, they will satisfy the supersymmetric algebra. Therefore we can conclude that in the $N \rightarrow \infty$ limit we have built a supersymmetric quantum model with infinite degrees of freedom and at the same time an infinite dimensional realization of the supersymmetric algebra.

Many other aspects deserve to be investigated in this model; we think the most interesting ones are:

a) the infinite volume limit for the "massless" theory and the possibility of a dynamical mass generation,

$\beta)$ the study of the supersymmetric "massive" theory.

The first problem is a difficult one. We would expect, as in the scalar theory, (Brydges et al. [11]) a dynamical generation of mass both for the Fermi and for the Bose fields which should allow us to control the infinite volume limit. In this case the analyticity in $\lambda$ would be lost, but, nevertheless, with a resummation one could possibly produce again convergent series for the Schwinger functions, at least for $\lambda$ real. At the present stage we are not able to extract any conclusion about the infinite volume theory.

Referring to $\beta$ ) it is clear, from our analysis of the effective potential of the theory, that if we add a bosonic and a fermionic mass term to the free part of the Lagrangian only, Theorem 1 is still valid and the theory is a perfectly defined field theory, but, unfortunately, the supersymmetry is explicitly broken. To construct the supersymmetric massive Sine-Gordon theory we have to add to the interaction a term proportional to $m \lambda \int_{A} \phi \sin \alpha \phi$ [see Eqs. (1.14), (1.15), and (1.16)]. To prove that such a theory exists should not be too hard but we are not able to control the convergence of the $\lambda$ series when this extra massive term is present.

Finally there are many other aspects of the Sine-Gordon theory which could be investigated as, for instance, the existence of infinite conserved currents; but we were more interested to look to this model as an explicit lab for testing Supersymmetry ideas than to see how much Supersymmetry modifies the properties of the scalar model. 


\section{Appendix A}

To construct the Euclidean Maiorana Spinors we follow Nicolai [7]. We have only to apply his results, mainly based on Osterwalder, Schrader [6] to the two dimensional case.

With our definitions of the $\gamma$ matrices (see Sect. 1) we have:

$$
\begin{aligned}
\psi_{M, \alpha}(x)= & (2 \pi)^{-1 / 2} \int d p\left[\frac{m}{\omega(p)}\right]^{1 / 2} e^{-i p x^{1}} \\
& \times\left[b^{+}(p) W_{(+), \alpha}^{*}(p) e^{-\omega(p) x^{0}}+b(-p) W_{(+), \alpha}(-p) e^{-\omega(p) x^{0}}\right], \\
\psi_{E, \alpha}(x)= & (2 \pi)^{-1} \int d^{2} p\left[\frac{e^{-i p x}}{\omega(p)}\right] \sum_{1}^{2}\left[B^{+}(p, j) V_{\alpha}^{j}(p)+B(-p, j) C_{\alpha p} \hat{V}_{\varrho}^{j}(p)\right],
\end{aligned}
$$

where

$$
W_{(+)}(p)=\frac{1}{2} \sqrt{\frac{\omega+m}{m}}\left(\begin{array}{c}
\frac{\omega+m-p}{\omega+m} \\
\frac{-i(\omega+m+p)}{\omega+m}
\end{array}\right),
$$

and the $b$ 's satisfy, as usual, the following anticommutation relations:

$$
\begin{gathered}
\left\{b(p), b^{+}\left(p^{\prime}\right)\right\}=\delta\left(p-p^{\prime}\right), \quad\left\{b(p), b\left(p^{\prime}\right)\right\}=\left\{b^{+}(p), b^{+}\left(p^{\prime}\right)\right\}=0 . \\
V_{\alpha}^{j}(p)=\left(S^{E}(-p) W^{j}(|p|)\right)_{\alpha} \\
\hat{V}_{\alpha}^{j}(p)=\left(\hat{W}^{j}(|p|)^{T} S^{E}(-p)^{-1}\right)
\end{gathered}
$$

where

$$
S^{E}(-p)=\frac{1}{\sqrt{2|p|\left(p_{0}+|p|\right)}}\left(\begin{array}{cc}
p_{0}+|p|+i p_{1} & 0 \\
0 & p_{0}+|p|-i p_{1}
\end{array}\right)
$$

and

$$
\begin{aligned}
& W^{(2,1)}(|p|)=(2 i)^{-1 / 2} \sqrt{i|p| \mp m}\left(\begin{array}{c}
1 \\
\pm i
\end{array}\right), \\
& \hat{W}^{(2,1)}(|p|)=(2 i)^{-1 / 2} \sqrt{i|p| \mp m}\left(\begin{array}{c} 
\pm i \\
1
\end{array}\right),
\end{aligned}
$$

where $|p|=\left(p_{0}^{2}+p_{1}^{2}\right)^{1 / 2}$ and the $B$ 's satisfy the anticommutation relations: $\left\{B(p, j), B^{+}\left(p^{\prime}, j^{\prime}\right)\right\}=\delta_{j, j^{\prime}} \delta\left(p-p^{\prime}\right), \quad\left\{B(p, j), B\left(p^{\prime}, j^{\prime}\right)\right\}=\left\{B^{+}(p, j), B^{+}\left(p^{\prime}, j^{\prime}\right)\right\}=0$.

\section{Appendix B}

From Sect. 1 the free Lagrangian density is proportional to $\Phi(x, \theta)\left(\mathscr{P}^{\alpha} \mathscr{P}_{\alpha}\right) \Phi(x, \theta)$ in the superfield formalism. Defining

$$
\delta \Phi=\xi \cdot Q \Phi=\left(-\xi_{2} Q_{1}+\xi_{1} Q_{2}\right) \Phi,
$$

it is easy to prove that, at the first order in $\xi$

$$
d \mathscr{L}=\delta \Phi\left(\mathscr{P}_{1} \mathscr{P}_{2}\right) \Phi+\Phi\left(\mathscr{P}_{1} \mathscr{P}_{2}\right) \delta \Phi
$$


is a total derivative. The same result holds for the interacting part of the Lagrangian. Passing to the fields $\phi, \psi$, and $F$ we get:

$$
\begin{aligned}
& \delta \phi=i\left(-\xi_{2} \psi_{1}+\xi_{1} \psi_{2}\right)=i(\xi \cdot \psi), \\
& \left.\begin{array}{l}
(\delta \psi)_{1}=-\left(\xi_{1} F-\xi_{2} \partial_{+} \phi\right) \\
(\delta \psi)_{2}=-\left(\xi_{2} F-\xi_{1} \partial_{-} \phi\right)
\end{array}\right\}(\delta \psi)_{\alpha}=-\left(\xi_{\alpha} F+\left(\gamma^{\mu} \xi\right)_{\alpha} \partial_{\mu} \phi\right), \\
& \delta F=i\left(\xi_{2} \partial_{+} \psi_{2}-\xi_{1} \partial_{-} \psi_{1}\right)=-\left(\xi \gamma^{\mu} \partial_{\mu} \psi\right) .
\end{aligned}
$$

The corresponding Euclidean transformations are:

$$
\begin{aligned}
\delta \phi_{E} & =i\left(-\xi_{2} \psi_{E_{1}}^{(1)}+\xi, \psi_{E_{2}}^{(1)}\right) \\
\left(\delta \psi_{E}^{(1)}\right)_{\alpha} & =-\left(-i \xi_{\alpha} F_{E}+\left(\partial^{E} \xi_{\alpha} \phi_{E}=\left\{\begin{array}{l}
i \xi_{1} F_{E}-i \xi_{2} \widetilde{\partial}_{+} \phi_{E} \\
i \xi_{2} F_{E}+i \xi_{1} \widetilde{\partial}_{-} \phi_{E}
\end{array}\right.\right.\right. \\
\delta F_{E} & =i\left(\xi_{2} \tilde{\partial}_{+} \psi_{E_{2}}^{(1)}+\xi_{1} \tilde{\partial}_{-} \psi_{E_{1}}^{(1)}=-\left(\xi \partial^{E} \psi\right),\right.
\end{aligned}
$$

where $\tilde{\partial}_{ \pm}=\partial_{0} \pm i \partial_{1}$.

The regularized version of Eqs. (2.8) is (we omit the index $E$ ):

$$
\begin{aligned}
& \phi^{[\leqq N]}(x)=\frac{1}{L^{2}} \sum_{p \in \frac{2 \pi}{L} Z^{2} ; p_{1} \neq 0} e^{i p x} \phi^{[\leqq N]}(p), \\
& \psi_{\alpha}^{[\leqq N]}(x)=\frac{1}{L^{2}} \sum_{p \in \frac{2 \pi}{L} Z^{2} ; p_{1} \neq 0} e^{i p x} \psi_{\alpha}^{[\leqq N]}(p), \\
& F^{[\leqq N]}(x)=\frac{1}{L^{2}} \sum_{p \in \frac{2 \pi}{L} Z^{2} ; p_{1} \neq 0} e^{i p x} F^{[\leqq N]}(p) .
\end{aligned}
$$

It is also immediate to write the corresponding expressions when $\Lambda$ is a cylinder. If we define the transformations:

$$
\begin{gathered}
\phi^{\prime[\leqq N]}=\phi^{[\leqq N]}+\delta \phi^{[\leqq N]}, \\
\psi_{\alpha}^{[\leqq N]}=\psi_{\alpha}^{[\leqq N]}+\delta \psi_{\alpha}^{[\leqq N]}, \quad \alpha=1,2, \\
\left.F^{[} \leqq N\right]=F^{[\leqq N]}+\delta F^{[\leqq N]},
\end{gathered}
$$

with $\delta \phi^{[\leqq N]}, \delta \psi_{\alpha}^{[\leqq N]}$, and $\delta F^{[\leqq N]}$ defined as in Eqs. (B.4) with the regularized fields everywhere, the regularized measure (6.8) is invariant under these transformations; the proof is just a matter of computation or the translation of the formal result plus the relations $(1.10)$.

Proof of Eq. (6.10). From the invariance under (B.6) of the regularized measure it follows that

$$
\left\langle\psi_{\beta}^{[\leqq N]}(x) \sin \alpha \phi^{[\leqq N]}(x)\right\rangle_{\lambda, \Lambda, N}=\left\langle\left(\psi_{\beta}^{[\leqq N]}+\delta \psi_{\beta}^{[\leqq N]}\right)(x) \sin \alpha\left(\phi^{[\leqq N]}+\delta \phi^{[\leqq N]}\right)(x)\right\rangle_{\lambda, \Lambda, N} .
$$

Choosing $\beta=1$, using the explicit expressions:

$$
\delta \phi=i\left(-\xi_{2} \psi_{1}^{(1)}+\xi_{1} \psi_{2}^{(1)}\right), \quad(\delta \psi)_{1}=-\left(\xi_{1} F-\xi_{2} \partial_{+} \phi\right),
$$


and remembering that $\xi_{1}$ and $\xi_{2}$ are independent Grassmann variables we get

$$
\begin{gathered}
-i \xi_{1} \int d^{2} x\left(\alpha\left\langle\psi_{1} \psi_{2} \cos \alpha \phi\right\rangle-\langle F \sin \alpha \phi\rangle\right)=0, \\
i \xi_{2} \frac{1}{\alpha} \int d^{2} x \partial_{+}\langle\cos \alpha \phi\rangle=0,
\end{gathered}
$$

where we omitted all the regularization indices. The first relation is exactly Eq. (5.11). Therefore Eq. (5.11) is proved and the proof of Theorem 2 is completed.

Acknowledgements. We thank G. Gallavotti for having drawn our attention to this model and also for many discussions and a crucial suggestion for the regularization of its lattice version; G. Jona Lasinio and P.K. Mitter for some interesting discussions. One of us (F.N.) wants to thank Arthur Jaffe for introducing him to the beauty of the Supersymmetry, K. Osterwalder for important discussions and for a very useful criticism on this work, and $\mathbf{M}$. Testa for some useful discussions and suggestions.

\section{References}

1. Witten, E.: Dynamical breaking of supersymmetry. Nucl. Phys. B 185, 513-554 (1981); Constraints on supersymmetry breaking. Nucl. Phys. B 202, 253-316 (1982)

2. Jaffe, A., Lesniewski, A., Weitsman, J.: Index of a family of Dirac operators on loop space. Commun. Math. Phys. 112, 75-88(1987); The two dimensional $N=2$ Wess-Zumino model on a cylinder. Commun. Math. Phys. 114, 147-165 (1988)

3. Benfatto, G., Cassandro, M., Gallavotti, G., Nicoló, F., Olivieri, E., Presutti, E., Scacciatelli, E.: On the ultraviolet stability in the Euclidean scalar field theories. Commun. Math. Phys. 71, $59(1980)$

4. Gallavotti, G.: Renormalization theory and ultraviolet stability for scalar fields via renormalization group methods, Rev. Mod. Phys. 57, 2 (1985)

Gallavotti, G., Nicolò, F.: Renormalization theory in four dimensional scalar fields (I), Commun. Math. Phys. 100, 545 (1985), Renormalization theory in four dimensional scalar fields (II), Commun. Math. Phys. 101, 247 (1985)

5. Wess, J., Bagger, J.: Supersymmetry and supergravity. Princeton, NJ: Princeton University Press

6. Osterwalder, K., Schrader, R.: Axioms for Euclidean Green's functions. I. Commun. Math. Phys. 31, 83 (1973); Axioms for Euclidean Green's functions. II. Commun. Math. Phys. 42, 281 (1973)

7. Nicolai, H.: A possible constructive approach to $\left(\text { Super- } \phi^{3}\right)_{4}$, (I), Nucl. Phys. B 140, 294 (1978)

8. Faddeev, L.D., Slavnov, A.A.: Gauge fields introduction to quantum theory. Menlo Park, CA: The Benjamin Cummings 1980

9. Wightman, A.S.: Cargèse Lectures, 1964. Levy, M. (ed.). New York: Gordon and Breach 1967

10. Cecotti, S., Girardello, L.: Functional measure, topology and dynamical supersymmetry breaking. Phys. Lett. 110 B, 39 (1983)

11. Brydges, D.: A rigorous approach to Debye screening in dilute classical Coulomb systems. Commun. Math. Phys. 58, 313 (1978)

Brydges, D., Federbush, P.: Debye screening. Commun. Math. Phys. 73, 197 (1980)

12. Gawedzki, K., Kupiainen, A.: Asymptotic freedom beyond perturbation theory. Les Houches 1984, Critical phenomena, random systems, gauge theories. Osterwalder, K., Stora, R. (eds.)

13. Fröhlich, J., Seiler, E.: The massive Thirring Schwinger model $\left(\mathrm{QED}_{2}\right)$ convergence of perturbation theory and particle structure. Helv. Phys. Acta 49, 889 (1976)

14. Benfatto, G.: An iterated Mayer expansion for the Yukawa gas. J. Stat. Phys. 41, 671 (1985)

15. Wilson, K.: New Developments in quantum field theory and statistical mechanics. Levy, M., Mitter, P.K. (eds.). New York, London: Plenum Press 1977 
16. Benfatto, G., Gallavotti, G., Nicolò, F.: On the massive Sine-Gordon equation in the first few regions of collapse. Commun. Math. Phys. 83, 387 (1982)

Nicolò, F.: On the massive Sine-Gordon equation in the higher regions of collapse. Commun. Math. Phys. 88, 681 (1983)

Nicolò, F., Renn, J., Steinmann, A.: The pressure of the two dimensional Coulomb gas at low and intermediate temperatures. Ann. Inst. H. Poincarè 144, 211 (1986)

Nicolò, F., Renn, J., Steinmann, A.: On the massive Sine-Gordon equation in all regions of collapse. Commun. Math. Phys. 105, 291 (1986)

17. Corwin, L., Ne'eman, Y., Sternberg, S.: Graded Lie algebras in mathematics and physics (Bose-Fermi symmetry), Rev. Mod. Phys. 47, 573 (1975)

Communicated by K. Gawedzki

Received September 13, 1988 